\title{
A New Method to Construct Secret Sharing Schemes Based on Linear Codes
}

\author{
Selda Çalkavur \\ Department of Mathematics, Kocaeli University, Kocaeli, Turkey
}

\begin{abstract}
Secret sharing is an important topic in cryptography and has applications in information security. The coding theory has been an important role in the constructing of secret sharing schemes. It is known that every linear code can be used to construct secret sharing schemes. So, we use the parity-check matrix of a linear code to construct secret sharing schemes based on linear codes. We also describe some techniques to recover the secret and determine the access structure of the new scheme. In this paper, we use the Massey's secret sharing scheme.
\end{abstract}

Key words: Linear code, parity-check matrix, secret sharing scheme, minimal codeword, minimal access set.

\section{Introduction}

Secret sharing has been a subject of study for over 30 years. Secret sharing schemes were explained by Blakley [3] and Shamir [13] in 1979. Then many constructions have been proposed. One of them is based on coding theory. Several authors have considered the construction of secret sharing schemes using error correcting codes [4, 7-9, 11, 16]. Massey used linear codes for secret sharing and mentioned the relationship between the access structure and the minimal codewords of the dual code of the underlying code $[8,16]$. But determining the minimal codewords is very hard for linear codes. Several authors have explained the minimal codewords for certain codes and characterized the access structures of secret sharing schemes based on their dual codes $[1,2,12$, 14].

Massey constructed a secret sharing scheme based on an $[n, k]$ - linear code $C$ using the generator matrix of this code. In this paper, we construct a secret sharing scheme based on $C^{\perp}$ using the parity-check matrix of an $[n, k]-$ linear code $C$.

This work is organized as follows. Section 2

Corresponding author: Selda Çalkavur, Assist. Prof. Dr., research fields: coding theory, cryptography. E-mail: selda.calkavur@kocaeli.edu.tr. reminds the necessary definitions. Section 3 explains a new method to construct secret sharing schemes based on linear codes and determines the access structure of this scheme.

\section{Definitions}

We shall describe a new method about how a secret sharing scheme can be construct based on linear codes. We begin with the necessary definitions.

\subsection{Codes and Secret Sharing}

Let $q$ be a prime power and denote the finite field of order $q$ by $F_{q}$ An $[n, k]$ - linear code $C$ over $F_{q}$ is a linear subspace in $\left(F_{q}\right)^{n}$, where $n$ is length of the code $C$ and $k$ is dimension of $C$. The dual code of $C$ is defined to be the set of those vectors $\left(F_{q}\right)^{n}$ which are orthogonal to every codeword of $C$ and is denoted by $C^{\perp} \cdot C^{\perp}$ is an $[n, n-k]-$ linear code.

Let $G=\left(g_{0}, g_{1}, \cdots, g_{n-1}\right)$ be a generator matrix of $[n, k]-$ code $C$, where $g_{0}, g_{1}, \cdots, g_{n-1}$ are column vectors of $G$. $G$ is a $k \times n$ matrix. There are several ways to use linear codes to construct secret sharing schemes. One of them is described by Massey. There are three parts in the Massey's secret sharing scheme: the secret which 
is element of $F_{q},(n-1)$ participants and a dealer.

Let $s \in F_{q}$ be the secret. The dealer chooses randomly a vector $u=\left(u_{0}, u_{1}, \cdots, u_{n-1}\right) \in\left(F_{q}\right)^{k}$ such that $s=u g_{0}$. The vector $u$ is the information vector [5]. So, the main question is "How is the secret shared between the participants?" and one of the important problems is "If the dealer lose the secret, how is it recovered?".

In this work, we use the following definitions in Ref. [10]:

The set $S=\left\{0 \leq i \leq n-1 \mid c_{i} \neq 0\right\}$ is called support of a vector $c=c_{1} c_{2} \ldots c_{n} \in\left(F_{q}\right)^{n}$. A codeword $c_{2}$ covers a codeword $c_{1}$ if the support of $c_{2}$ contains that of $c_{1}$.

A minimal codeword $c$ is a codeword which covers just only its scalar multiples.

The access structure of a secret sharing scheme is the set of all minimal access sets.

\section{A New Method to Construct Secret Sharing Schemes Based on Linear Codes}

Massey constructed a secret sharing scheme based on an $[n, k]-$ linear code $C$ using the generator matrix $G$ of $C$. We know that the codewords of the code $C$ can obtain by the parity-check matrix of $C$. Thus, we use the parity-check matrix of an $[n, k]$-code $C$. So, we construct a secret sharing scheme based on an $[n, n-k]-$ linear code $C^{\perp}$ using the parity-check matrix $H$ of $C$.

Let $H=\left(h_{0}, h_{1}, \ldots, h_{n-1}\right)$ be a parity-check matrix of an $[n, k]$ - linear code $C$, where $h_{0}, h_{1}, \ldots, h_{n-1}$ are column vectors of $H . H$ is also the generator matrix of the dual code $C^{\perp} H$ is a $(n-k) \times n$ matrix. In this paper, we always assume that no column vector of any parity-check matrix is the zero vector.

Now we construct a secret sharing scheme based on linear codes by a new method.

Let $F_{q}$ be the secret space, $s$ be the secret and $K_{1}, K_{2}, \ldots, K_{n-1}$ be $(n-1)$ participants. The dealer randomly chooses a vector $u=\left(u_{0}, \quad u_{1}, \ldots, \quad u_{n-k-1}\right) \in\left(F_{q}\right)^{n-k} \quad$ such that $s=u h_{0}$. Such a vector $u$ can be chosen in $q^{n-k-1}$ ways. The secret shared between the participants as follows.

The dealer computes the corresponding codeword

$$
t=\left(t_{0}, t_{1}, \ldots, t_{n-1}\right)=u H
$$

and gives $t_{i}$ to participant $K_{i}$ as the share for each $i \geq 1$. Since $t_{0}=u h_{0}=s$, the set $T=\left\{t_{i_{1}}, t_{i_{2}}, \ldots, t_{i_{m}}\right\}$ is a set of shares, where $1 \leq i_{1}<\ldots<i_{m} \leq n-1$ and $1 \leq m \leq n-1$.

In the following lemma, we give a necessary and sufficient condition to recover the secret in the secret sharing scheme based on the dual code $C^{\perp}$.

Lemma 3.1. Let $H$ be a parity-check matrix of an $[n, k]$ - linear code $C$ over $F_{q}$. In the secret sharing scheme based on an $[n, n-k]-$ dual code $C^{\perp}$ of the code $C$, determines the secret if and only if $h_{0}$ is a linear combination of $h_{1}, h_{2}, \ldots, h_{n-1}$ are column vectors of $H$.

Proof. Suppose that $h_{0}=\left(h_{11}, h_{21}, \ldots, h_{(n-k) 1}\right)^{T}$ is a linear combination of $h_{1}, h_{2}, \ldots, h_{n-1}$.

$$
\begin{gathered}
h_{0}=\alpha_{1} h_{1}+\alpha_{2} h_{2}+\ldots+\alpha_{n-1} h_{n-1}, \quad\left(\alpha_{i} \in F_{q},\right. \\
1 \leq i \leq n-1)
\end{gathered}
$$

This means

$$
\begin{aligned}
& h_{11}=\alpha_{1} h_{12}+\ldots+\alpha_{n-1} h_{1 n} \\
& h_{21}=\alpha_{1} h_{22}+\ldots+\alpha_{n-1} h_{2 n} \\
& \ldots \ldots \ldots \ldots \ldots \ldots \ldots \ldots \ldots \ldots \ldots \ldots \\
& h_{(n-k) 1}=\alpha_{1} h_{(n-k) 2}+\ldots+\alpha_{n-1} h_{(n-k) n} .
\end{aligned}
$$

In expression

$$
s=u_{0} h_{11}+u_{1} h_{21}+\ldots+u_{n-k-1} h_{(n-k) 1}
$$

if it is written of values $h_{11}, h_{21}, \ldots, h_{(n-k) 1}$, then the secret $s$ is determined.

Suppose that the secret $s$ is determined. Since $s=u h_{0}$, that is

$s=u_{0} h_{11}+u_{1} h_{21}+\ldots+u_{n-k-1} h_{(n-k) 1}$.

This means $h_{0}=\left(h_{11}, h_{21}, \ldots, h_{(n-k) 1}\right)^{T}$ is known. By definition of the parity-check matrix $H$ is written by 


$$
\begin{aligned}
& h_{11}=\alpha_{1} h_{12}+\ldots+\alpha_{n-1} h_{1 n} \\
& h_{21}=\alpha_{1} h_{22}+\ldots+\alpha_{n-1} h_{2 n} \\
& \ldots \ldots \ldots \ldots \ldots \ldots \ldots \ldots \ldots \ldots \ldots \ldots \ldots \ldots \ldots \ldots \ldots \ldots \ldots \ldots \ldots \ldots \ldots \ldots \ldots \ldots \ldots \\
& h_{(n-k) 1}=\alpha_{1} h_{(n-k) 2}+\ldots .
\end{aligned}
$$

Therefore it is obtained that $h_{0}$ is a linear combination of $h_{1}, h_{2}, \ldots, h_{n-1}$.

By the following theorem we explain a new necessary and sufficient condition to recover the secret in the secret sharing scheme based on $C^{\perp}$.

Theorem 3.2. Let $H$ be a parity-check matrix of an $[n, k]-$ linear code $C$ over $F_{q}$. In the secret sharing scheme based on $C^{\perp}$, a set of shares $T=\left\{t_{i_{1}}, t_{i_{2}}, \ldots, t_{i_{m}}\right\} \quad\left(1 \leq i_{1}<\ldots<i_{m} \leq n-1\right.$ and $1 \leq m \leq n-1)$ determines the secret if and only if there is

a

codeword $v=\left(1,0, \ldots, c_{i_{1}}, 0, \ldots, c_{i_{m}}, 0, \ldots, 0\right)$ in the code $C$, where $c_{i_{j}} \neq 0$ for at least one $j$.

Proof. Suppose that $v \in C$. Then we should show $h_{0}$ is a linear combination of $h_{i_{1}}, h_{i_{2}}, \ldots, h_{i_{m}}$ $\left(1 \leq i_{1}<\ldots<i_{m} \leq n-1\right.$ and $\left.1 \leq m \leq n-1\right)$. By definition of $C$ if $v \in C$, then

$$
v H^{T}=0 \text {. }
$$

So

$$
\begin{aligned}
& h_{11}+h_{1 i_{1}} c_{i_{1}}+\ldots+h_{1 i_{m}} c_{i_{m}}=0 \\
& h_{21}+h_{2 i_{1}} c_{i_{1}}+\ldots+h_{2 i_{m}} c_{i_{m}}=0 \\
& \ldots \ldots \ldots \ldots \ldots \ldots \ldots \ldots \ldots \ldots \ldots \ldots \ldots \ldots \ldots \ldots \ldots \ldots \ldots \ldots \ldots \ldots \ldots \ldots \ldots \ldots \ldots \\
& h_{(n-k) 1}+h_{(n-k) i_{1}} c_{i_{1}}+\ldots .+h_{(n-k) i_{m}} c_{i_{m}}=0
\end{aligned}
$$

Therefore

$$
\begin{aligned}
& h_{11}=-\left(h_{1 i_{1}} c_{i_{1}}+\ldots+h_{1 i_{m}} c_{i_{m}}\right) \\
& h_{21}=-\left(h_{2 i_{1}} c_{i_{1}}+\ldots+h_{2 i_{m}} c_{i_{m}}\right) \\
& \ldots \ldots \ldots \ldots \ldots \ldots \ldots \ldots \ldots \ldots \ldots \ldots \ldots \ldots . . . \ldots \ldots \ldots \ldots \ldots \ldots \ldots \ldots \\
& h_{(n-k) 1}=-\left(h_{(n-k) i_{1}} c_{i_{1}}+\ldots+h_{(n-k) i_{m}} c_{i_{m}} .\right.
\end{aligned}
$$

This means $h_{0}=\left(h_{11}, h_{21}, \ldots, h_{(n-k) 1}\right)^{T}$ is a linear combination of $h_{i_{1}}, h_{i_{2}}, \ldots, h_{i_{m}}$, where $1 \leq i_{1}<\ldots<i_{m} \leq n-1$ and $1 \leq m \leq n-1$. That is

$$
h_{0}=\sum_{j=1}^{m} x_{j} h_{i_{j}} .
$$

Then the secret is recovered by computing

$$
s=\sum_{j=1}^{m} x_{j} t_{i_{j}} .
$$

Now suppose that in the secret sharing scheme based on $C^{\perp}$, a set of shares $T=\left\{t_{i_{1}}, t_{i_{2}}, \ldots, t_{i_{m}}\right\}$ $\left(1 \leq i_{1}<\ldots<i_{m} \leq n-1\right.$ and $\left.1 \leq m \leq n-1\right)$ determines the secret. We should show that $v \in C$. Suppose that $v \notin C$. Then the proof is very easy.

From Theorem 3.2, it is clear that in the secret sharing scheme based on $C^{\perp}$ there is one-to-one correspondence between the set of minimal access sets and the set of minimal codewords of the code $C$ whose first coordinate is 1 . To determine the access structure of secret sharing scheme, we need to determine only the set of minimal codewords in $C$ whose first coordinate is 1 .

We know that to recover the secret in a secret sharing scheme is very important. To recover the secret, it has to be known the access structure of secret sharing scheme is very difficult. We also know that the access structure of a secret sharing scheme is the set of all minimal access sets. So, we have to determine the number of minimal access sets in the secret sharing scheme.

Now we give a theorem about the number of minimal access sets in the secret sharing scheme based on a linear code $C$ over $F_{q}$.

Theorem 3.3. Let $C$ be an $[n, k]$-code over $F_{q}$ and $H=\left(h_{0}, h_{1}, \ldots, h_{n-1}\right)$ be its parity-check matrix, where all $h_{i}(1 \leq i \leq n-1)$ are nonzero. If each nonzero codeword of $C^{\perp}$ is minimal, then in the secret sharing scheme based on $C$, there are altogether $q^{n-k-1}$ minimal access sets.

Proof. Since all column vectors of the parity-check matrix $H$ are nonzero, $h_{0} \neq 0$. It is written $q^{n-k-1}$ different codewords for all vector $u$ such that $u \in\left(F_{q}\right)^{n-k}$. Thus the inner product $u h_{0}$ 
takes on each element of $F_{q}$ exactly $q^{n-k-1}$ times when $u$ ranges over all elements of $\left(F_{q}\right)^{n-k} \cdot u h_{0}$ is equal to multiplication the first column of the parity-check matrix $H$ by $u$. At result it is determined the first coordinate of the codeword. If $u=0$, then there are $q^{n-k-1}$ codewords whose first coordinate is zero. So, there are altogether

$$
q q^{n-k-1}=q^{n-k}
$$

codewords in $C^{\perp}$ whose first coordinate is any element of $F_{q}$. Hence there are altogether

$$
q^{n-k}-q^{n-k-1}
$$

codewords in $C^{\perp}$ whose first coordinate is nonzero. Since each nonzero codeword is a minimal vector, a codeword covers another one if and only if they are multiples of each other. Hence the total number of minimal codewords is

$$
\frac{q^{n-k}-q^{n-k-1}}{q-1}=q^{n-k-1},
$$

which is the number of minimal access sets.

Example 3.4. We consider the binary [7, 4]-code $C$. For this code the parameters are $n=7, k=4$. A generator matrix $G$ and a parity-check matrix $H$ of $[7,4]$-code $C$ can be given as follows.

$$
\begin{aligned}
& G=\left[\begin{array}{lllllll}
1 & 0 & 0 & 0 & 0 & 1 & 1 \\
0 & 1 & 0 & 0 & 1 & 0 & 1 \\
0 & 0 & 1 & 0 & 1 & 1 & 0 \\
0 & 0 & 0 & 1 & 1 & 1 & 1
\end{array}\right] \\
& H=\left[\begin{array}{lllllll}
0 & 1 & 1 & 1 & 1 & 0 & 0 \\
1 & 0 & 1 & 1 & 0 & 1 & 0 \\
1 & 1 & 0 & 1 & 0 & 0 & 1
\end{array}\right]
\end{aligned}
$$

There are $2^{4}=16$ codewords in the code $C$. These codewords are

$\left\{\begin{array}{l}1101000 \\ 0110100 \\ 0011010 \\ 0001101 \\ 1000110 \\ 0100011 \\ 1010001 \\ 0101110 \\ 0010111 \\ 1001011 \\ 1100101 \\ 1110010 \\ 0111001 \\ 1011100 \\ 0000000 \\ 1111111\end{array}\right.$.

The dual code $C^{\perp}$ of the code $C$ is a linear $[7,3]$ - code. So $\left|C^{\perp}\right|=2^{3}=8$. The codewords of $C^{\perp}$ are

$\left\{\begin{array}{l}0101110 \\ 0010111 \\ 1001011 \\ 1100101 \\ 1110010 \\ 0111001 \\ 1011100 \\ 0000000\end{array}\right.$.

Now we find the supports of nonzero codewords in $C^{\perp}$.

$\{2,4,5,6\},\{3,5,6,7\},\{1,4,6,7\},\{1,2,5,7\},\{1$, $2,3,6\},\{2,3,4,7\},\{1,3,4,5\}$.

None of these sets does not cover each other. So, all of the nonzero codewords in $C^{\perp}$ are minimal. By Theorem 3.3, if each nonzero codeword of $C^{\perp}$ is 
minimal, then in the secret sharing scheme based on $C$ there are altogether $q^{n-k-1}$ minimal access sets. Thus, there are

$$
2^{7-4-1}=4
$$

minimal access sets. By Theorem 3.2, we know that in the secret sharing scheme based on the dual code $C^{\perp}$, there is one-to-one correspondence between the set of minimal access sets and the set of minimal codewords of the code $C$ whose first coordinate is 1 . So, we can say that in the secret sharing scheme based on the code $C$, there is one-to-one correspondence between the set of minimal access sets and the set of minimal codewords of the dual code $C^{\perp}$ whose first coordinate is 1 . Therefore the minimal access sets are

$\{1001011\},\{1100101\},\{1110010\},\{1011100\}$.

These are the set of minimal codewords in $C^{\perp}$ whose first coordinate is 1 .

\section{Conclusion}

In this work, proposed a new method to construct secret sharing schemes based on linear codes. So, used the parity-check matrix $H$ of an $[n, k]$ - linear code $C . H$ is also the generator matrix for an $[n, n-k]-$ dual code $C^{\perp}$. Therefore constructed based on an $[n, n-k]-$ dual code $C^{\perp}$ using the parity-check matrix $H$ of $C$. In the constructed scheme, explained the necessary and sufficient conditions to determine the secret and calculated the number of minimal access sets in the secret sharing scheme based on $C$ while each nonzero codeword of $C^{\perp}$ is minimal. In final section, explained in the secret sharing scheme based on the dual code $C^{\perp}$ there is one-to-one correspondence between the set of minimal access sets and the set of minimal codewords of the code $C$ whose first coordinate is 1 .

\section{Acknowledgement}

The author would like to thank Jin Yuan and Cunsheng Ding for their valuable paper which is called "Secret Sharing Schemes from Three Classes of Linear Codes".

\section{References}

[1] Ashikmin, A., Barg, A., and Cohen, G. 1995. "Variations on Minimal Codewords in Linear Codes." In Proc. Applied Algebra, Algebraic Algorithms and Error-Correcting Codes (AAECC 1995) (Lecture Notes in Computer Science). Berlin, Germany: Springer-Verlag, Vol. 948, 96-105.

[2] Ashikmin, A., and Barg, A. Sep. 1998. "Minimal Vectors in Linear Codes." IEEE Trans. Inf. Theory 44 (5): 2010-7.

[3] Blakley, G. R. Jun. 1979. "Safeguarding Cryptographic Keys.” In Proc. 1979 National Computer Conf., New York, 313-7.

[4] Ding, C., Kohel, D., and Ling, S. 2000. "Secret Sharing with a Class of Ternary Codes." Theor. Comp. Sci. 246: 285-98.

[5] Dougherty, S. T., Mesnager, S., and Sole, P. May 2008. "Secret Sharing Schemes Based on Self-dual Codes." Information Theory Workshop, ITW 08. IEEE, 338-42.

[6] Hill, R. 1986. A First Course in Coding Theory. Oxford: Oxford University.

[7] Karnin, E. D., Greene, J. W., and Hellman, M. E. Jan. 1983. "On Secret Sharing Systems." IEEE Trans. Inf. Theory IT-29 (1): 35-41.

[8] Massey, J. L. Aug. 1993. "Minimal Codewords and Secret Sharing." In Proc. 6th Joint Swedish-Russian Workshop on Information Theory, Mölle, Sweden, 276-9.

[9] Okada, K., and Kurusowa, K. May 2000. "MDS Secret Sharing Scheme Secure against Cheaters." IEEE Trans. Inf. Theory 46 (3): 1078-81.

[10] Özadam, H., Özbudak, F., and Sayg1, Z. December 2007. "Secret Sharing Schemes and Linear Codes." Information Security Cryptology Conference with International Participation, Proceedings, 101-6.

[11] Piepryzk, J. X., and Zhang, M. 2003. "Ideal Threshold Schemes from MDS Codes." In Information Security and Cryptology-Proc. of ICISC 2002 (Lecture Notes in Computer Science). Berlin, Germany: Springer-Verlag, Vol. 2587, 269-79.

[12] Renvall, A., and Ding, C. 1996. "The Access Structure of Some Secret Sharing Schemes." In Information Security and Privacy (Lecture Notes in Computer Science). Berlin, Germany: Springer-Verlag, Vol. 1172, 67-78.

[13] Shamir, A. 1979. "How to Share a Secret." Commun. Assoc. Comp. Mach. 22: 612-3.

[14] Yuan, J., and Ding, C. Dec. 2002. "Secret Sharing Schemes from Two Weight Codes." In Proc. R. C. Bose Centenary Symp. Discrete Mathematics and Applications, Kolkata, India.

[15] Yuan, J., and Ding, C. Jan. 2006. "Secret Sharing Schemes from Three Classes of Linear Codes." IEEE 
Trans. on Inf. Theory 52 (1): 206-12.

[16] Massey, J. L. 1995. "Some Applications of Coding
Theory." Cryptography, Codes and Ciphers: Cryptography and Coding IV, 33-47. 